\title{
Receiver design for the uplink of base station cooperation systems employing SC-FDE modulations
}

Filipe Casal Ribeiro ${ }^{1,2^{*}}$, Rui Dinis ${ }^{1,3},{\text { Francisco } \text { Cercas }^{1,2} \text { and Adão Silva }}^{1,4}$

\begin{abstract}
The presented paper considers the uplink transmission in base station (BS) cooperation schemes where mobile terminals (MTs) in adjacent cells share the same physical channel. We consider single-carrier with frequency-domain equalization (SC-FDE) combined with iterative frequency-domain receivers based on the iterative block decision feedback equalization (IB-DFE). We study the quantization requirements when sending the received signals, from different MTs, at different BSs to a central unit that performs the separation of different MTs using iterative frequency-domain receivers. Our performance results show that a relatively coarse quantization, with only 4 bits in the in-phase and quadrature components of the complex envelope already allows close-to-optimum macro-diversity gains, as well as an efficient separation of the transmitted signals associated with each MT.
\end{abstract}

Keywords: BS cooperation; SC-FDE; IB-DFE; Quantization

\section{Introduction}

It is expected that the demand for wireless services will continue to increase in the near and medium term, calling for more capacity and putting more and more pressure on the usage of radio resources. Future spectral efficiency improvements will be focused on interferencereducing techniques that require the cooperation of network elements [1], since the point-to-point link capacities are already close to the fundamental Shannon limit [2]. Conventional cellular systems adopt different frequencies at different cells, with frequency reuse factors of 3 , or even more. Clearly, the overall system's spectral efficiency and capacity are conditioned by the frequency reuse factor, typically decreasing linearly with it. Since the spectrum is a scarce and expensive resource in wireless systems, it would be desirable to design systems operating in universal frequency reuse (i.e., with frequency reuse factor 1). Multicell cooperation is a promising solution to mitigate interference between different cells, to improve the system's fairness and to increase capacity [3-7]. For these reasons, it is already under study in LTE

${ }^{*}$ Correspondence: casalribeiro.filipe@gmail.com

1 IT - Instituto de Telecomunicações, 1049-001 Lisboa, Portugal

2ISCTE - Instituto Universitário de Lisboa, 1649-026 Lisboa, Portugal

Full list of author information is available at the end of the article
[8], namely, under the so-called coordinated multi-point concept which although not included in current releases, will probably be specified for future ones.

In conventional cellular architectures, different cells are regarded as separate entities and each mobile terminal (MT) is assigned to a given cell and, consequently, a given base station (BS). The MT transmits its signals to the corresponding BS, and when this signal is received by another BS, it is regarded as interference. In BS cooperation architectures [9], the signals between different MTs and BSs are collected and processed by a central processing unit (CPU), so as to perform the user separation and/or interference mitigation. In fact, BS cooperation allows not only universal frequency reuse but also substantial macro-diversity effects, significantly improving the overall performance of wireless systems, as well as the coverage and power requirements associated with each individual link. The signal separation in the downlink transmission (i.e., the link from the BSs to the MTs) of BS cooperation schemes is usually achieved by appropriate pre-processing schemes [10-12]. In the uplink transmission (i.e., the link from the MTs to the BSs), the overall signals received by different $\mathrm{BSs}$ (with contributions from all MTs) are sent to the CPU that performs the signal separation, to extract the data blocks transmitted by each

\section{Springer}

(c) 2015 Ribeiro et al: licensee Springer. This is an Open Access article distributed under the terms of the Creative Commons Attribution License (http://creativecommons.org/licenses/by/4.0), which permits unrestricted use, distribution, and reproduction in any medium, provided the original work is properly credited. 
MT, before sending them to the corresponding BS [3]. These BS cooperation schemes involve interference mitigation, allowing the use of the same physical channel by MTs in adjacent cells, which means that the overall system capacity can be significantly improved.

It is well known that block transmission techniques, combined with frequency-domain processing, are choice candidates for broadband wireless systems. These techniques include orthogonal frequency division multiplexing (OFDM) [13] and single-carrier with frequency domain equalization (SC-FDE) [14], which have similar overall signal processing requirements and achievable performance. However, the receiver complexity is higher for SC-FDE, and the transmitter complexity is higher for OFDM. If we also take into account that the envelope fluctuations of single-carrier signals are much lower than the envelope fluctuations of OFDM signals with the same constellations, SC-FDE is clearly preferable for the uplink transmission while OFDM is interesting mainly for the downlink transmission $[15,16]$.

In this paper, we consider the uplink of broadband wireless systems with BS cooperation architectures with the employment of SC-FDE modulation schemes. MTs in adjacent cells can share the same physical channel and the signals received by a given BS are digitalized, through appropriate sampling and quantization procedures. The corresponding bits are sent to the central unit. The separation of the signals associated with different MTs is performed using iterative frequency-domain receivers based on the iterative block decision feedback equalization (IBDFE) concept [17-19]. Due to its processing nature, the IB-DFE can be considered as a frequency-domain low complexity turbo equalizer [20-22]. Hence, the IB-DFE does not need the channel decoder output at the feedback loop. Nevertheless, turbo equalizers based on IB-DFE schemes can be conceived [23]. The quantization requirements for the signals received at a given BS are studied in detail.

This paper is organized as follows: in Section 2, we describe the BS cooperation scenario considered in this paper and Section 3 is concerned with the receiver design. A set of performance results is presented in Section 4, and Section 5 concludes the paper.

Throughout the paper, we will adopt the following notations: bold letters denote vectors and matrices; $\mathbf{x}^{*}, \mathbf{x}^{T}$, and $\mathbf{x}^{H}$ denote complex conjugate, transpose, and Hermitian (complex conjugate transpose) of $\mathbf{x}$, respectively. $\mathbf{I}_{N}$ denotes a $N \times N$ identity matrix, and $\mathbf{e}_{p}$ is an appropriate column vector with 0 in all positions except the $p$ th position that is 1 . The expectation of $x$ is denoted by $\mathbb{E}[x]$.

\section{System characterization}

The considered system (shown in Figure 1) is characterized by partially overlapping cells, where each one is associated with a certain BS. For the sake of simplicity, we considered only single-antenna transmitters and receivers. However, our detection procedure and the corresponding performance analysis could easily be extended to multi-antenna scenarios. In the considered scheme, $P$ MTs share the same physical channel (i.e., the information from all MTs is transmitted at the same frequency band) and, in general, there are $R \geq P$ BSs which receive the MTs signals and can efficiently cooperate to improve the system's performance. Although sometimes, we can have $R<P$, in general, we need at least $R=P$ to be able to separate $P$ users. By adopting $R>P$, it is possible to have macro-diversity effects, allowing further performances. Typically, in conventional systems, one BS only performs the detection of the signals of its own MTs, interpreting the information from other MTs as interference, that is, ignoring it. Contrarily to conventional architectures, this paper considers a BS cooperation system where the overall signals received at each BS are quantized and sent to a central processing unit that performs the separation of the different transmitted signals and then sends them to the corresponding BS.

It is assumed perfect synchronization and channel estimation. This can be obtained with the help of appropriate training blocks and/or pilots [24-26]. The channel estimation segments are similar to other SC-FDE schemes [27].

An SC-FDE scheme is employed by each MT and the data block associated with the $p$ th MT $(p=1,2, \ldots, P)$ is $\left\{s_{n, p} ; n=0,1, \ldots, N-1\right\}$, where constellation symbol $s_{n, p}$ is selected from the data, according to a given mapping rule (e.g., a quadrature phase-shift keying (QPSK) constellation with Gray mapping). The corresponding frequencydomain block is $\left\{S_{k, p} ; k=0,1, \ldots, N-1\right\}=$ DFT $\left\{s_{n, p} ; n=0,1, \ldots, N-1\right\}$. As with other block transmission techniques, an appropriate cyclic prefix is appended to each data block. The useful time-domain received block, i.e., after removing the samples associated with the cyclic prefix, at the $r$ th BS is $\left\{y_{n}^{(r)} ; n=0,1, \ldots, N-1\right\}$ $(r=1,2, \ldots, R)$, and the corresponding frequencydomain block is $\left\{Y_{k}^{(r)} ; k=0,1, \ldots, N-1\right\}=\mathrm{DFT}$ $\left\{y_{n}^{(r)} ; n=0,1, \ldots, N-1\right\}$. Clearly, we have

$$
y_{n}^{(r)}=\sum_{p=1}^{P} \xi_{p, r} \sum_{n^{\prime}} s_{n-n^{\prime}, p} h_{n^{\prime}, p}^{(r)}+v_{n}^{(r)},
$$

where $h_{n, p}^{(r)}$ denotes the channel impulse response between the $p$ th MT and the $r$ th BS, for the $n$th time-domain component. $v_{n}^{(r)}$ indicates the channel noise at the $r$ th BS and the $n$th time-domain component. The $\xi_{p, r}$ parameter is a weighting factor that accounts for the combined effects 


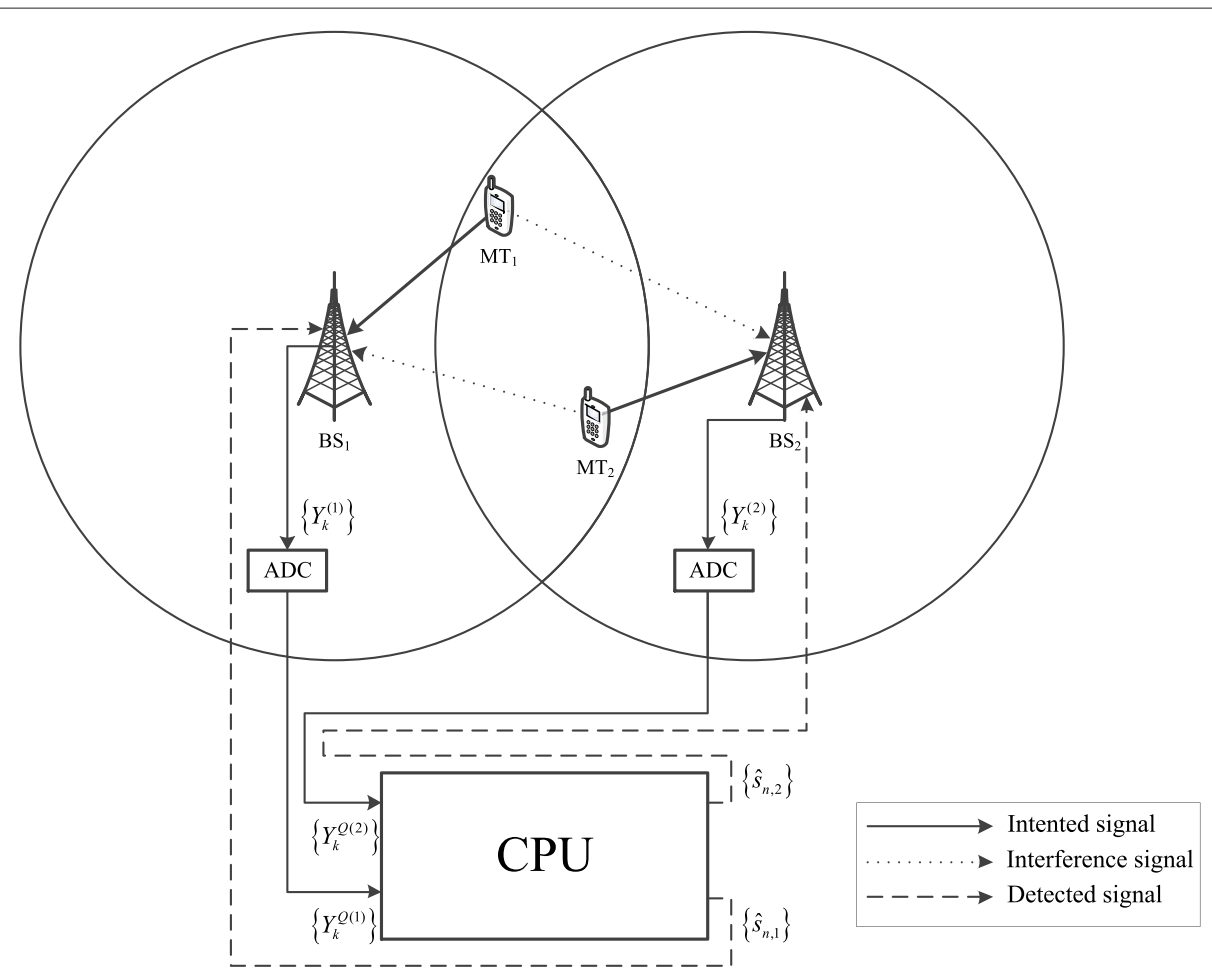

Figure 1 Adopted cellular scenario for 2 MTs and 2 BSs.

of power control and propagation loss effects (the average received power associated with the $p$ th MT at the $r$ th BS is $\left|\xi_{p, r}\right|^{2}$ ). In conventional block transmission schemes, the cyclic prefix is required to be longer than the overall impulse response, including channel effects and transmit and receive filters. However, in BS cooperation schemes, it might be necessary to have a slightly longer cyclic prefix to account for different propagation times between MTs and BSs, since the useful part of each block should overlap. This means that if the cyclic prefix is long enough, it can be shown that [19]:

$$
y_{n}^{(r)}=\sum_{p=1}^{P} \xi_{p, r} S_{n, p} \circledast h_{n, p}^{(r)}+v_{n}^{(r)},
$$

with $\circledast$ denoting the cyclic convolution in $n$. Consequently, in the frequency-domain, we have:

$$
Y_{k}^{(r)}=\sum_{p=1}^{P} \xi_{p, r} S_{k, p} H_{k, p}^{(r)}+N_{k}^{(r)},
$$

with $H_{k, p}^{(r)}$ and $N_{k}^{(r)}$ corresponding to the frequencydomain of $h_{n, p}^{(r)}$ and $v_{n}^{(r)}$, respectively, considering a normalized channel with $\sum_{n=1}^{N} \mathbb{E}\left[\left|h_{n, p}^{(r)}\right|^{2}\right]=1$. For severely time-dispersive channels, with rich multipath propagation, the received samples $y_{n}^{(r)}$ can be regarded as samples of a zero-mean complex Gaussian process, i.e., $y_{n}^{(r)} \sim$ $\mathcal{C N}\left(0,2 \sigma_{y}^{(r)^{2}}\right)$, with:

$$
\begin{aligned}
2 \sigma_{y}^{(r)^{2}} & =\mathbb{E}\left[\left|y_{n}^{(r)}\right|^{2}\right]=\sum_{p=1}^{P}\left|\xi_{p, r}\right|^{2} \mathbb{E}\left[\left|s_{n, p}\right|^{2}\right]+\mathbb{E}\left[\left|v_{n}^{(r)}\right|^{2}\right] \\
& =\sum_{p=1}^{P}\left|\xi_{p, r}\right|^{2} 2 \sigma_{s}^{2}+2 \sigma_{v}^{2},
\end{aligned}
$$

where $\sigma_{s}^{2}=\mathbb{E}\left[\left|\operatorname{Re}\left\{s_{n}\right\}\right|^{2}\right]=\mathbb{E}\left[\left|\operatorname{Im}\left\{s_{n}\right\}\right|^{2}\right]$ and $\sigma_{v}^{2}=$ $\mathbb{E}\left[\left|\operatorname{Re}\left\{v_{n}\right\}\right|^{2}\right]=\mathbb{E}\left[\left|\operatorname{Im}\left\{v_{n}\right\}\right|^{2}\right]$. The received signals at the $r$ th BS, $\left\{y_{n}^{(r)} ; n=0,1, \ldots, N-1\right\}$, are quantized ${ }^{\mathrm{a}}$, leading to $y_{n}^{Q(r)}$, with

$$
y_{n}^{Q(r)}=f_{Q}\left(\frac{\operatorname{Re}\left\{y_{n}^{(r)}\right\}}{\sigma_{y}^{(r)}}\right) \sigma_{y}^{(r)}+j f_{Q}\left(\frac{\operatorname{Im}\left\{y_{n}^{(r)}\right\}}{\sigma_{y}^{(r)}}\right) \sigma_{y}^{(r)},
$$

where $f_{Q}(\cdot)$ denotes the quantization characteristic. Due to the Gaussian nature of $y_{n}^{(r)}$, it can be shown that the quantized signals can be decomposed as the sum of uncorrelated useful and distortion terms (see Appendix), i.e.:

$$
y_{n}^{Q(r)} \approx \alpha y_{n}^{(r)}+d_{n}^{(r)},
$$


with $d_{n}^{(r)}$ denoting the quantization noise term and $\alpha$ computed as described in the Appendix. Clearly, $\frac{\operatorname{Re}\left\{y_{n}^{(r)}\right\}}{\sigma_{y}^{(r)}}$ and $\frac{\operatorname{Im}\left\{y_{n}^{(r)}\right\}}{\sigma_{y}^{(r)}}$ are uncorrelated $\mathcal{C N}(0,1)$ random variables. Since the nonlinear distortion component when the input $\mathcal{C N}(0,1)$ has variance $\sigma_{d}^{2}$ (see Appendix), we have:

$$
2 \sigma_{d}^{(r)^{2}}=\mathbb{E}\left[\left|d_{n}^{(r)}\right|^{2}\right]
$$

and

$$
\sigma_{d}^{(r)^{2}}=\sigma_{y}^{(r)^{2}} \sigma_{d}^{2}
$$

where $\sigma_{d}^{2}$ denotes the variance of the distortion term when the input is $\mathcal{C N}(0,1)$, which can be computed as described in the Appendix. We can also define a signal-toquantization noise ratio (SQNR) as:

$$
\text { SQNR }=\frac{\mathbb{E}\left[\left|\alpha y_{n}^{(r)}\right|^{2}\right]}{\mathbb{E}\left[\left|d_{n}^{Q(r)}\right|^{2}\right]} .
$$

The SQNR is a function of the number of quantization levels $2^{m}$, with $m$ denoting the number of bits required for the real and imaginary parts of each quantized sample, and the type of quantizer. For the sake of simplicity, we will consider only uniform quantizers in this paper, i.e., $f_{Q}(\cdot)$ has $2^{m}$ levels equally spaced between the saturation levels $-A_{M}$ and $A_{M}$. Figure 2 shows the impact of the normalized saturation level $A_{M} / \sigma_{y}^{(r)}$ and the number of quantization bits $m$ on SQNR. Actually, the number of quantization bits is $2 m$, since we need $m$ bits for the real part and $m$ bits for the imaginary part, according to (5). For comparison, we include the conventional SQNR that assumes uniform distribution for the quantization noise, therefore neglecting saturation effects, and the signalto-distortion ratio (SDR) for an ideal clipping, therefore neglecting the quantization error. Clearly, the SDR is accurate for small quantization steps $\left(A_{M} / \sigma_{y}^{(r)}<<1\right)$, and the uniform approximation, for the quantization noise, is reasonable for high values of $A_{M} / \sigma_{y}^{(r)}$. Moreover, there is an optimum normalized saturation level for each value of $m$, since the quantizer's saturation becomes too frequent if $A_{M} / \sigma_{y}^{(r)}$ is small and the quantization interval becomes too high when $A_{M} / \sigma_{y}^{(r)}$ is high. In this paper, we always assume the optimum saturation level for each value of $m$.

The frequency-domain block, associated with the quantized signal at the $r$ th BS, is $\left\{Y_{k}^{Q(r)} ; k=0,1, \ldots, N-1\right\}=$ DFT $\left\{y_{n}^{Q(r)} ; n=0,1, \ldots, N-1\right\}$, where:

$$
Y_{k}^{Q(r)} \approx \alpha Y_{k}^{(r)}+D_{k}^{Q(r)} \approx \alpha \sum_{p=1}^{P} \xi_{p, r} S_{k, p} H_{k, p}^{(r)}+N_{k}^{\mathrm{Tot}(r)} .
$$

$N_{k}^{\text {Tot }(r)}=\alpha N_{k}^{(r)}+D_{k}^{(r)}$ denotes the overall noise, from the transmitted and quantized signals, in which:

$$
2 \sigma_{D}^{(r)^{2}}=\mathbb{E}\left[\left|D_{k}^{(r)}\right|^{2}\right]=N \mathbb{E}\left[\left|d_{n}^{(r)}\right|^{2}\right]
$$

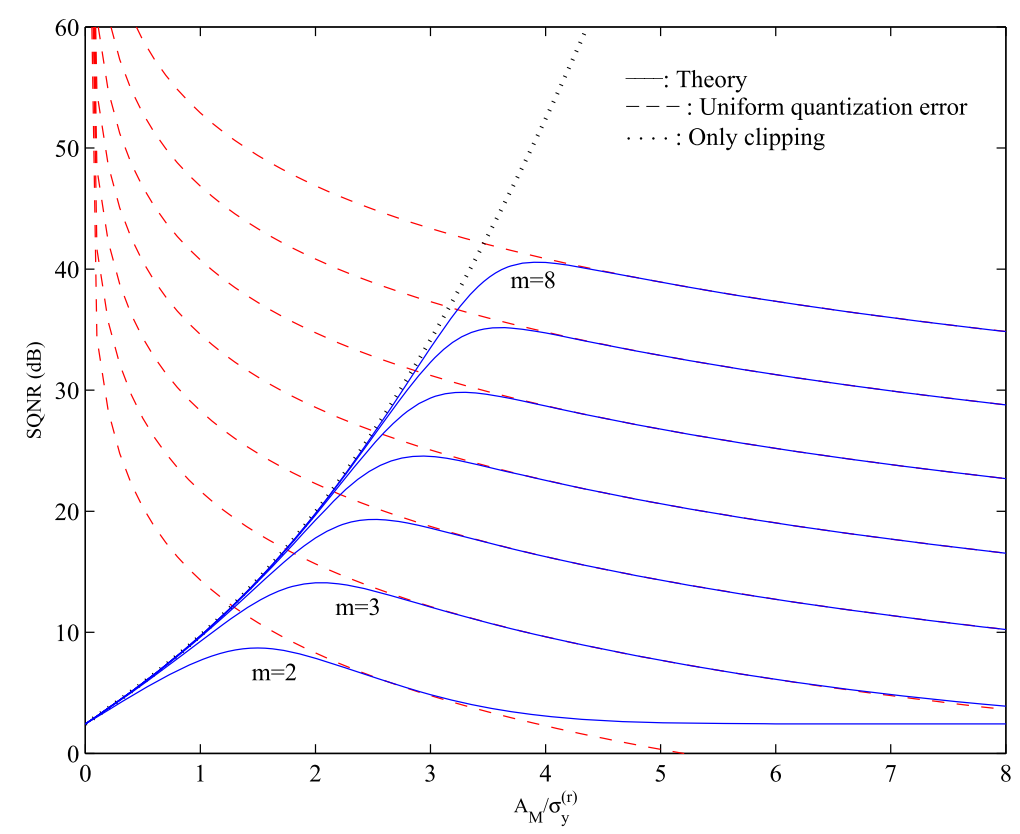

Figure 2 SQNR as a function of the normalized saturation level $A_{M} / \sigma_{y}^{(r)}$ and the number of quantization bits $m$. 
since we considered the IDFT and DFT definitions $d_{n}^{(r)}=$ $\frac{1}{N} \sum_{k=0}^{N-1} D_{k}^{(r)} e^{j 2 \pi \frac{k n}{N}}$, and $D_{k}^{(r)}=\sum_{n=0}^{N-1} d_{n} e^{-j 2 \pi \frac{k n}{N}}$, respectively. Moreover, we have:

$$
2 \sigma_{N}^{\operatorname{Tot}(r)^{2}}=\mathbb{E}\left[\left|N_{k}^{\operatorname{Tot}(r)}\right|^{2}\right]=2 \sigma_{D}^{(r)^{2}}+2 \sigma_{N}^{2}|\alpha|^{2} .
$$

In matrix format, (10) is equivalent to

$$
\mathbf{Y}_{k}^{Q}=\mathbf{H}_{k}^{T} \mathbf{S}_{k}+\alpha \mathbf{N}_{k}+\mathbf{D}_{k}
$$

with $\mathbf{Y}_{k}^{Q}=\left[Y_{k}^{Q(1)}, \ldots, Y_{k}^{Q(R)}\right]^{T}, \mathbf{S}_{k}=\left[S_{k, 1}, \ldots, S_{k, P}\right]^{T}$, $\mathbf{N}_{k}=\left[N_{k}^{(1)}, \ldots, N_{k}^{(R)}\right]^{T}, \mathbf{D}_{k}=\left[D_{k}^{(1)}, \ldots, D_{k}^{(R)}\right]^{T}$,

$$
\mathbf{H}_{k}^{T}=\left[\begin{array}{ccc}
H_{k, 1}^{e q^{(1)}} & \ldots & H_{k, P}^{e q^{(1)}} \\
\vdots & \ddots & \vdots \\
H_{k, 1}^{e q^{(R)}} & \ldots & H_{k, P}^{e q^{(R)}}
\end{array}\right] \text {, }
$$

and $H_{k, p}^{e q^{(r)}}=\alpha \xi_{p, r} H_{k, p}^{(r)}$.

\section{Receiver design}

In this section, the receiver design is presented concerning BS cooperation schemes. For this purpose, an iterative frequency-domain receiver based on the IB-DFE concept [19] is considered, as illustrated in Figure 3, allowing an efficient separation of the signals associated with different MTs, using the same physical channel that is able to take full advantage of macro-diversity effects. Figure 3 shows the different mechanisms in the iterative process (with the variables being defined starting in (16)), from the received data blocks after the quantization process to symbols estimation on each iteration. Ideally, we should sort out the MTs according to their overall power, given by:

$$
\sum_{k=0}^{N-1} \sum_{r=1}^{R}\left|\xi_{p, r} H_{k, p}^{(r)}\right|^{2}
$$

and to detect the MTs from the larger to the smaller, in terms of overall power ${ }^{b}$. However, our results show that our iterative receiver is highly robust to the detection order, provided that the number of iterations is high enough. In fact, the main advantage of a proper detection order is that we typically can slightly reduce the number of the required iterations for best performance. For each iteration, we detect all MTs in a successive way, using the most updated estimates of the transmitted data symbols, associated with each MT to cancel the corresponding residual interference (see Figure 4). Therefore, our receiver can be regarded as an iterative successive interference cancelation (SIC) scheme. However, as with conventional IB-DFE receivers, we take into account the reliability of the data estimates associated with MTs (as well as interference cancelation) for each detection procedure.

The complexity of our receiver is essentially conditioned by the pair FFT/IFFT for each user and each iteration, as well as the need to solve a system of $R$ equations, for every frequency of each user and each iteration.

At the $i$ th iteration, the estimated symbols associated with the $p$ th $\mathrm{MT}\left\{\hat{s}_{n, p} ; n=0,1, \ldots, N-1\right\}$ are the hard decisions of the time-domain detector

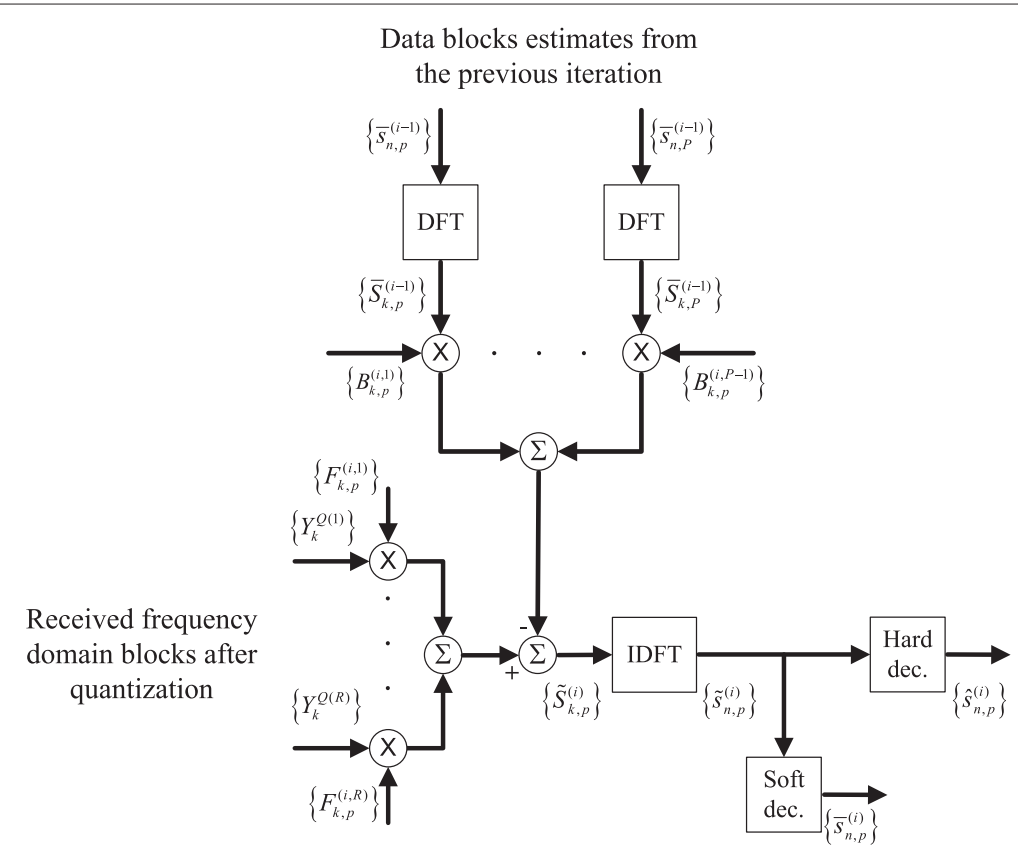

Figure 3 IB-DFE receiver design. 


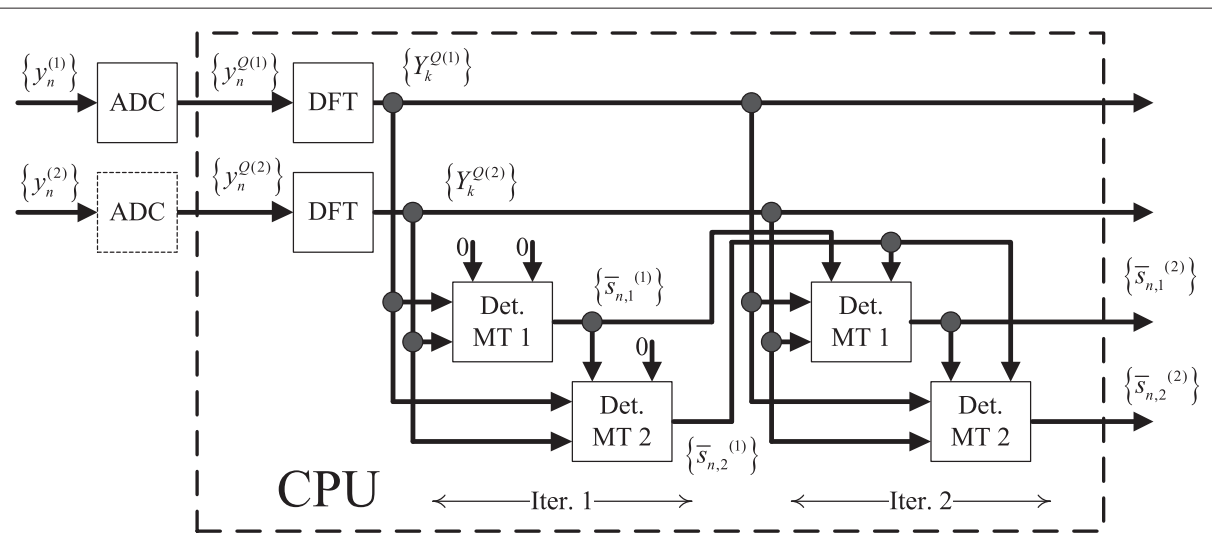

Figure 4 Iterative receiver structure for $P=2$ MTs and $R=2$ cooperating BSs with quantization.

output $\left\{\tilde{s}_{n, p} ; n=0,1, \ldots, N-1\right\}=\operatorname{IDFT}\left\{\tilde{S}_{k, p} ; k=\right.$ $0,1, \ldots, N-1\}$, where $\tilde{S}_{k, p}$ is given by

$$
\tilde{S}_{k, p}=\mathbf{F}_{k, p}^{T} \mathbf{Y}_{k}^{Q}-\mathbf{B}_{k, p}^{T} \overline{\mathbf{S}}_{k, p}
$$

with $\mathbf{F}_{k, p}^{T}=\left[F_{k, p}^{(1)}, \ldots, F_{k, p}^{(R)}\right]^{T}$ denoting the feedforward coefficients. $\mathbf{B}_{k, p}^{T}=\left[B_{k, p}^{(1)}, \ldots, B_{k, p}^{(P)}\right]^{T}$ denotes the feedback coefficients and vector $\overline{\mathbf{S}}_{k, p}$ is given by $\overline{\mathbf{S}}_{k, p}=\left[\bar{S}_{k, 1}, \ldots, \bar{S}_{k, p-1}, \bar{S}_{k, p}, \ldots, \bar{S}_{k, P}\right]^{T}$, where block $\left\{\bar{S}_{k, p} ; k=0,1, \ldots, N-1\right\}$ is the DFT of the block of timedomain average values conditioned to the detector output $\left\{\bar{s}_{n, p} ; n=0,1, \ldots, N-1\right\}$, for user $p$ at a given iteration. Clearly, the elements of $\overline{\mathbf{S}}_{k, p^{\prime}}$ are associated with the current iteration for the MTs already detected $\left(p^{\prime}<p\right)$ and associated with the previous iterations for the MT that is being detected, and also to the MTs still not detected in this iteration.

For normalized QPSK constellations, i.e., $s_{n, p}= \pm 1 \pm j$, the average values $\bar{s}_{n, p}$ are given by [28]:

$$
\bar{s}_{n, p}=\tanh \left(\frac{L_{n, p}^{R e}}{2}\right)+j \tanh \left(\frac{L_{n, p}^{\operatorname{Im}}}{2}\right),
$$

where

$$
\begin{aligned}
& L_{n, p}^{R e}=\frac{2}{\sigma_{n, p}^{2}} \operatorname{Re}\left\{\tilde{s}_{n, p}\right\}, \\
& L_{n, p}^{I m}=\frac{2}{\sigma_{n, p}^{2}} \operatorname{Im}\left\{\tilde{s}_{n, p}\right\},
\end{aligned}
$$

and

$$
\sigma_{n, p}^{2}=\frac{1}{2 N} \sum_{n^{\prime}=0}^{N-1}\left|\tilde{s}_{n^{\prime}, p}-s_{n^{\prime}, p}\right|^{2} \simeq \frac{1}{2 N} \sum_{n^{\prime}=0}^{N-1}\left|\tilde{s}_{n^{\prime}, p}-\hat{s}_{n^{\prime}, p}\right|^{2} .
$$

This means that we replace the transmitted symbols by the hard decision estimates in the computation of the $\sigma_{n, p}^{2}$, with its error being negligible for a low bit error rate (BER) [28]. The hard decisions associated with the symbol $s_{n, p}$ are $\hat{s}_{n, p}=\operatorname{sign}\left(\operatorname{Re}\left\{\tilde{s}_{n, p}\right\}\right)+j \operatorname{sign}\left(\operatorname{Im}\left\{\tilde{s}_{n, p}\right\}\right)$.

For a given iteration and the detection of the $p$ th MT, the receiver is characterized by coefficients $\mathbf{F}_{k, p}$ and $\mathbf{B}_{k, p}$ $(k=0,1, \ldots, N-1)$. These coefficients are selected to minimize the BER performance. For a QPSK constellation with Gray mapping, the BER is given by:

$$
P_{e} \simeq Q\left(\sqrt{\frac{1}{\theta_{p}}}\right)
$$

where $Q(x)$ denotes the well known Gaussian error function and:

$$
\theta_{p}=\frac{1}{N^{2}} \sum_{k=0}^{N-1} \Theta_{k, p}
$$

with

$$
\begin{array}{r}
\Theta_{k, p}=\mathbb{E}\left[\left|\tilde{S}_{k, p}-S_{k, p}\right|^{2}\right]= \\
\mathbb{E}\left[\left|\mathbf{F}_{k, p}^{T} \mathbf{Y}_{k}^{Q}-\mathbf{B}_{k, p}^{T} \overline{\mathbf{S}}_{k, p}-S_{k, p}\right|^{2}\right]
\end{array}
$$

designating the mean squared error (MSE) on the frequency-domain samples $\tilde{S}_{k, p}$ [29]. The processing of $\Theta_{k, p}$ concerns the evaluation of the optimum values of coefficients $\mathbf{F}_{k, p}$ and $\mathbf{B}_{k, p}$, and to do so, the minimization of $\Theta_{k, p}$ is required.

It can be shown that $\bar{S}_{k, p} \simeq \rho_{p} \hat{S}_{k, p}$ [30,31], with $\hat{S}_{k, p}$ denoting the frequency-domain samples associated with the symbols' hard decision. Moreover, $\hat{S}_{k, p} \approx \rho_{p} S_{k, p}+$ $\Delta_{k, p}$ [32], which means that $\bar{S}_{k, p} \approx \rho_{p}^{2} S_{k, p}+\rho_{p} \Delta_{k, p}$, and $\mathbb{E}\left[\left|\bar{S}_{k, p}\right|^{2}\right]=\left|\rho_{p}\right|^{2} \mathbb{E}\left[\left|\hat{S}_{k, p}\right|^{2}\right]=\left|\rho_{p}\right|^{2} \mathbb{E}\left[\left|S_{k, p}\right|^{2}\right]$. On the other hand, $\Delta_{k, p}=\left[\Delta_{k, 1}, \ldots, \Delta_{k, P}\right]^{T}$, is a mean zero error vector for $P$ MTs, with its elements uncorrelated to $S_{k, p}$ and between them, meaning that $\mathbb{E}\left[\left|\Delta_{k, p}\right|^{2}\right]=$ 
$\left(1-\left|\rho_{p}\right|^{2}\right) \mathbb{E}\left[\left|S_{k, p}\right|^{2}\right]$. In matrix format, we have $\overline{\mathbf{S}}_{k} \simeq$ $\mathbf{P}^{2} \mathbf{S}_{k}+\mathbf{P} \boldsymbol{\Delta}_{k}$ and $\mathbf{P}=\operatorname{diag}\left(\rho_{1}, \ldots, \rho_{P}\right)$, with correlation coefficients:

$$
\rho_{p}=\frac{\mathbb{E}\left[\hat{s}_{n, p} s_{n, p}^{*}\right]}{\mathbb{E}\left[\left|s_{n, p}\right|^{2}\right]}
$$

designating a measure of the estimates reliability associated with the $i$ th iteration. It can be shown that, for QPSK constellations, we have $\rho_{p}=1-2 P_{e}$ [30], which can be approximately given by:

$$
\rho_{p} \approx \frac{1}{2 N} \sum_{n=0}^{N-1}\left(\left|\rho_{n, p}^{R e}\right|+\left|\rho_{n, p}^{\operatorname{Im}}\right|\right),
$$

with

$$
\rho_{n, p}^{R e(i)}=\tanh \left(\frac{\left|L_{n, p}^{R e(i)}\right|}{2}\right),
$$

and

$$
\rho_{n, p}^{\operatorname{Im}(i)}=\tanh \left(\frac{\left|L_{n, p}^{\operatorname{Im}(i)}\right|}{2}\right) .
$$

By expanding the square in (22) and noting that the noise and data components are uncorrelated and have zero mean, it can be easily shown that $\Theta$ is given by:

$$
\begin{array}{r}
\Theta=\mathbf{F}^{H} \mathbf{R}_{\mathbf{Y} Q} \mathbf{F}+\mathbf{B}^{H} \mathbf{R}_{\overline{\mathbf{S}}, \overline{\mathbf{S}}} \mathbf{B}+ \\
\mathbf{R}_{S}-2 \operatorname{Re}\left\{\mathbf{F}^{H} \mathbf{R}_{\mathbf{Y}, S_{p}}\right\}+ \\
2 \operatorname{Re}\left\{\mathbf{B}^{H} \mathbf{R}_{\overline{\mathbf{S}}, S_{p}}\right\}-2 \operatorname{Re}\left\{\mathbf{B}^{H} \mathbf{R}_{\mathbf{S},{ }_{Y},} \mathbf{F}\right\} .
\end{array}
$$

For the sake of simplicity, the dependence on the subcarrier and user indexes, with the exception of the $S_{p}$ factor, were dropped from in (26) and following equations. The different correlation matrices of (26) are as follows:

$$
\begin{aligned}
& \mathbf{R}_{Y^{Q}}=\mathbb{E}\left[\mathbf{Y}^{Q^{*}} \mathbf{Y}^{Q^{T}}\right]=|\alpha|^{2} \mathbf{H}^{H} \mathbf{R}_{S} \mathbf{H}+\mathbf{R}_{N^{T o t}} \\
& \mathbf{R}_{\overline{\mathbf{S}}, \overline{\mathbf{S}}}=\mathbb{E}\left[\overline{\mathbf{S}}^{*} \overline{\mathbf{S}}^{T}\right]=\mathbf{P}^{2} \mathbf{R}_{S} \\
& \mathbf{R}_{\mathbf{Y}^{Q}, S_{p}}=\mathbb{E}\left[\mathbf{Y}^{Q^{*}} S_{p}\right]=\alpha \mathbf{H}^{H} \mathbf{R}_{S} \mathbf{e}_{p} \\
& \mathbf{R}_{\overline{\mathbf{S}}, S_{p}}=\mathbb{E}\left[\overline{\mathbf{S}}^{*} S_{p}\right]=\mathbf{P}^{2} \mathbf{R}_{S} \mathbf{e}_{p} \\
& \mathbf{R}_{\overline{\mathbf{S}}, \mathbf{Y}}=\mathbb{E}\left[\overline{\mathbf{S}}^{*} \mathbf{Y}^{Q}\right]=\alpha \mathbf{P}^{2} \mathbf{R}_{S} \mathbf{H},
\end{aligned}
$$

with $\mathbf{R}_{S}=\mathbb{E}\left[\mathbf{S}^{*} \mathbf{S}^{T}\right]=2 \sigma_{S}^{2} \mathbf{I}_{P}$ and $\mathbf{R}_{N^{T o t}}=$ $\mathbb{E}\left[\mathbf{N}^{\text {Tot }^{*}} \mathbf{N}^{\text {Tot }}{ }^{T}\right]=|\alpha|^{2} \mathbf{R}_{N}+\mathbf{R}_{D}$, being the correlation matrices of $\mathbf{S}$ and $\mathbf{N}^{\text {Tot }}$, respectively. $\mathbf{R}_{N}=2 \sigma_{N}^{2} \mathbf{I}_{R}$ and $\mathbf{R}_{D}=2 \operatorname{diag}\left(\sigma_{D}^{(1)^{2}}, \sigma_{D}^{(2)^{2}}, \ldots, \sigma_{D}^{(R)^{2}}\right)$ are the correlations matrices for the channel noise and quantization noise, respectively, and $\sigma_{S}^{2}$ and $\sigma_{N}^{2}$ denote the symbol's and noise's variance, in the frequency domain, respectively.

Clearly, the bit error probability will be minimized if we minimize the MSE at each subcarrier $\Theta_{k, p}$. In order to obtain the minimization of the MSE, we subject it to the condition

$$
\gamma_{p}=\frac{1}{N} \sum_{k=0}^{N-1} \sum_{r=1}^{R} F_{k, p}^{(r)} H_{k, p}^{e q(r)}=1,
$$

and apply the gradient of the Lagrange function to (22). Hence, the Lagrange function is defined as

$$
J=\Theta_{k, p}+\lambda\left(\gamma_{p}-1\right),
$$

where the optimum coefficients $\mathbf{F}_{k, p}$ and $\mathbf{B}_{k, p}$ being the solution for the system of equations:

$$
\left\{\begin{array}{l}
\nabla_{\mathbf{F}} J=0 \\
\nabla_{\mathbf{B}} J=0 \\
\nabla_{\lambda} J=0 .
\end{array}\right.
$$

Therefore:

$$
\left\{\begin{aligned}
& \nabla_{\mathbf{F}} J=0 \Leftrightarrow \mathbf{R}_{\mathbf{Y} Q} \mathbf{F}-\mathbf{R}_{\mathbf{Y}^{Q}, S_{p}}-\mathbf{R}_{\overline{\mathbf{S}} \mathbf{Y} Q}^{H} \mathbf{B}+\lambda \mathbf{H}^{H} \mathbf{e}_{p}=0 \\
& \nabla_{\mathbf{B}} J=0 \Leftrightarrow \mathbf{R}_{\overline{\mathbf{S}}, \overline{\mathbf{S}}} \mathbf{B}+\mathbf{R}_{\overline{\mathbf{S}}, S_{p}}-\mathbf{R}_{\overline{\mathbf{S}}, \mathbf{Y}^{Q}} \mathbf{F}=0 \\
& \nabla_{\lambda} J=0 \Leftrightarrow \gamma_{p}=1 .
\end{aligned}\right.
$$

After some straightforward manipulations, we obtain:

$$
\mathbf{F}=\kappa \Lambda \mathbf{H}^{H} \mathbf{e}_{p}
$$

and

$$
\mathbf{B}=\alpha \mathbf{H F}-\mathbf{e}_{p},
$$

with

$$
\Lambda=\left(\mathbf{H}^{H}\left(\mathbf{I}_{P}-\mathbf{P}^{2}\right) \mathbf{H}+\mathbf{R}_{N^{T o t}} \mathbf{R}_{S}^{-1}|\alpha|^{-2}\right)^{-1},
$$

and $\kappa$ selected to ensure that $\gamma_{p}=1$, in order to have a normalized FDE with $\mathbb{E}\left[\tilde{s}_{n, p}\right]=s_{n, p}$.

It should be pointed out that when we ignore the quantization effects, $\mathbf{R}_{N^{\mathrm{Tot}}}=\mathbf{R}_{N}$ and $\alpha=1$. In practice, $\alpha \simeq 1-2 Q\left(\frac{A_{M}}{\sigma_{y}^{(r)}}\right)$ [33], which means that for practical quantizers, $\alpha \simeq 1$ and using $\alpha=1$ has a negligible impact on the receiver's performance.

\section{Performance results}

In this section, we present a set of performance results considering the proposed iterative frequency-domain receivers for the uplink of BS cooperation schemes employing SC-FDE modulations with the corresponding quantization impact. The blocks associated with each MT have $N=256$ data symbols, selected from a QPSK constellation under a Gray mapping rule, plus an appropriate cyclic prefix. We considered a multipath channel with 64 symbol-spaced taps and uncorrelated Rayleigh fading 
on the different multipath components (similar results were observed for other channels with rich multipath propagation). The channels between different transmitting and receiving antennas are assumed uncorrelated. We have perfect synchronization and channel estimation. It is assumed that the useful part of the blocks transmitted by different MTs arrive at each BS simultaneously. In practice, this could be accomplished by employing extended cyclic prefixes, with duration longer than the maximum overall channel impulse response plus the difference between the maximum and minimum propagation delay between MTs and BSs, provided that we have accurate channel estimates. The real and imaginary parts of the complex envelope of the signals received by each BS are sampled and quantized by a uniform quantizer with $2^{m}$ levels (i.e., $m$ bits) and sent to the central unit. Throughout this section, the theoretical results were obtained with the analytical MSE-based approach described in the previous section. Initially, a single-MT case $(P=1)$ is considered. Figure 5 illustrates the BER performance when we have a single BS, as in conventional point-to-point communications, and when we have two BSs $(R=2)$, as in a macro-diversity scenario. All the links considered between the MT and the BSs are characterized by $\xi_{p, r}=0$ $\mathrm{dB}$. From the corresponding results, it is clear that the proposed analytical approach is very precise, especially regarding the first iteration (linear FDE). When the subsequent iterations are considered, that is, when the Gaussian approximation is accurate, the difference between the simulated and analytical approaches vary only in a few tenths of dB. As expected, the BER performance improves with the number of iterations, and it is clearly better when there is a macro-diversity scenario $(R=2 \mathrm{BSs})$.

Let us consider now a BS cooperation scenario with $P=2$ MTs and $R=2 \mathrm{BSs}$. The power associated with the different links $\xi_{p, r}$ can be characterized by the matrix:

$$
\Xi=\left[\begin{array}{ll}
\xi_{1,1} & \xi_{1,2} \\
\xi_{2,1} & \xi_{2,2}
\end{array}\right]=\left[\begin{array}{ll}
-3 & -3 \\
-3 & -3
\end{array}\right](\mathrm{dB})
$$

where both MTs are at the cell's edge and perfect average power control, corresponding to a scenario with strong interference between MTs at both BSs. The BER performance for this scenario is depicted in Figures 6 and 7, for the first and second MTs, respectively.

The BER performance is excellent, with the corresponding values close to the MFB after four iterations, which means that the proposed receiver is able to efficiently separate the MTs while taking advantage of the signal contributions associated with a given MT at each BS. The simulated results are very accurate for the first MT that is detected, with a slight difference between analytical and simulated results, although this difference is always below $1 \mathrm{~dB}$ and most of the time even lower, proving right accuracy for our analytical approach.

Let us now consider the case where we employ the quantization process. Figures 8 and 9 represent the BER performance in a conventional scenario $(P=R=1)$ for iterations 1 and 4 , respectively. Here, samples are quantized with $m=2,3,4,5$, and $\infty$ bits, in which $m=\infty$ indicates that no quantization is performed. The power from link from the MT and the BS is $\xi=0 \mathrm{~dB}$. In both

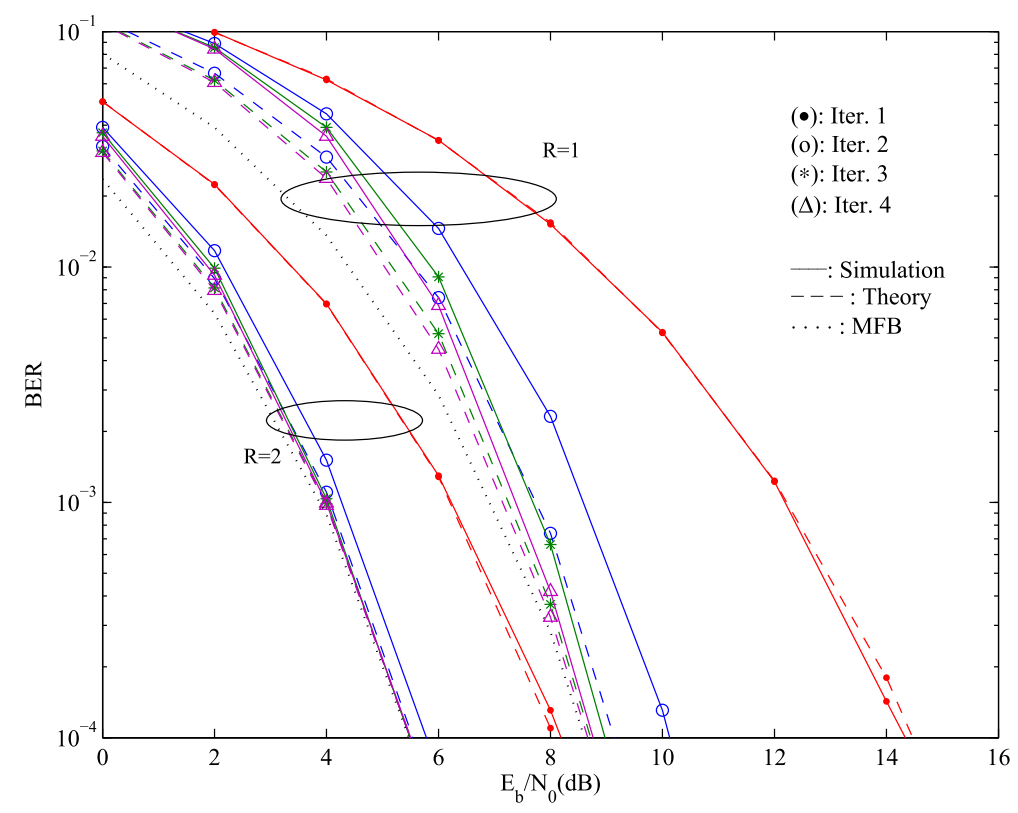

Figure 5 BER performance for $P=1 \mathrm{MT}, R=1 \mathrm{BS}$ (without macro-diversity) and $P=1 \mathrm{MT}, R=2 \mathrm{BSs}$ (with macro-diversity). 


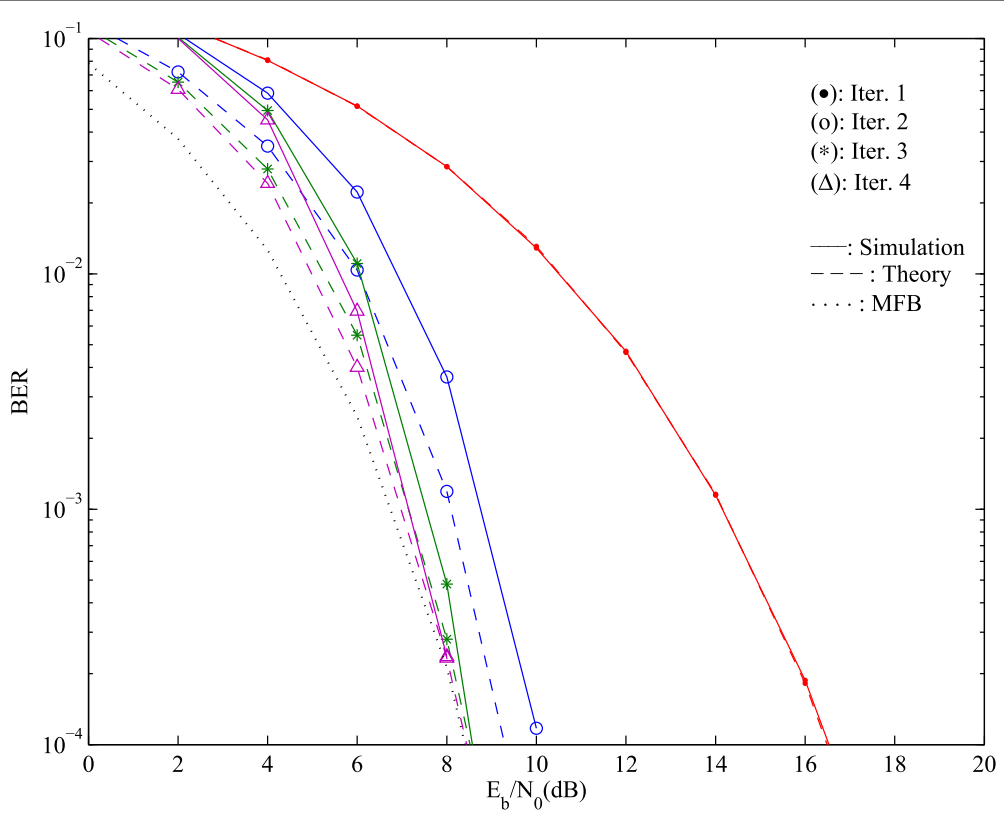

Figure 6 BER performance for a strong interference BS cooperation scenario with $P=2$ MTs and $R=2$ BSs (first MT).

figures, a conventional receiver is presented (denoted as 'Conv. $\mathrm{Rx}$ '), where we do not take into account the characteristics of the quantization noise, and an optimized receiver (indicated as 'Opt. $\mathrm{Rx}$ '), where the quantization noise is taken into account.

Clearly, with $m$ above 4 , the degradation due to quantization effects is negligible, and below 4, the performance degradation can be considerable. On the first iteration, the degradation considering the conventional receiver is higher, with a significant difference between performances even when $m=4$. Ultimately, for the fourth iteration, the main advantage in considering the optimum receiver is when the quantization is employed with low values of $m$, such as $m=2$ bits.

For a macro-diversity scenario and samples quantized with $m=3$ and 4 bits, Figures 10 and 11, respectively,

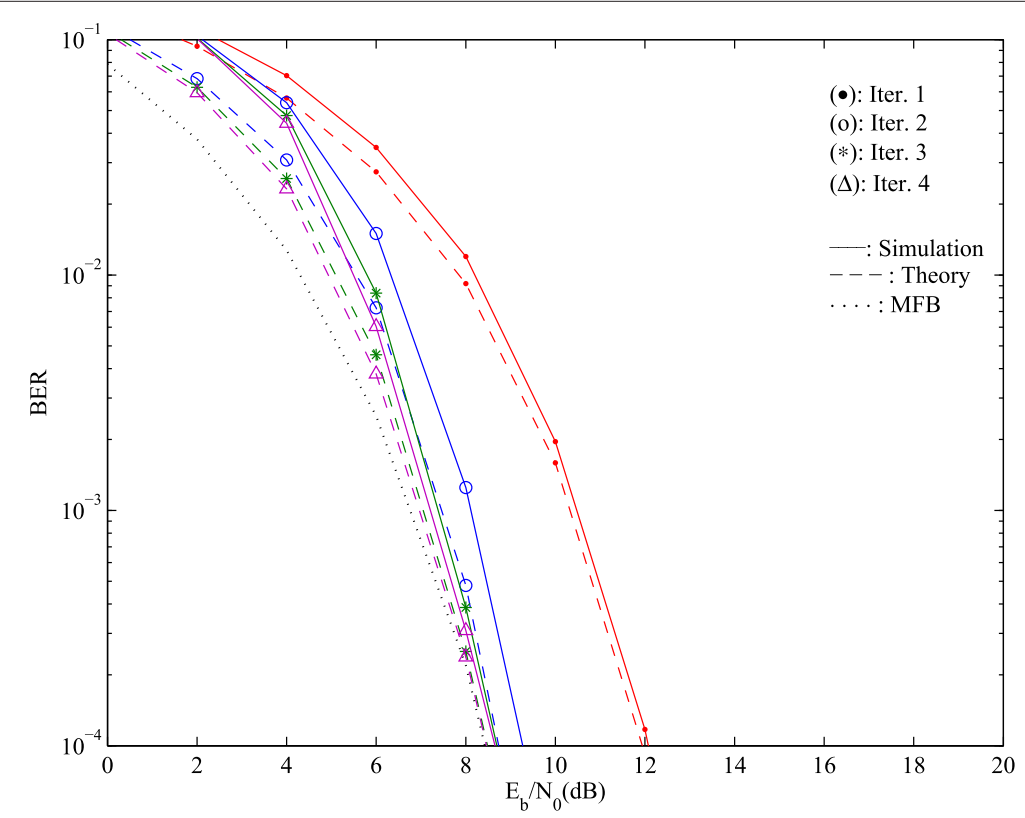

Figure 7 BER performance for a strong interference BS cooperation scenario with $P=2$ MTs and $R=2$ BSs (second MT). 


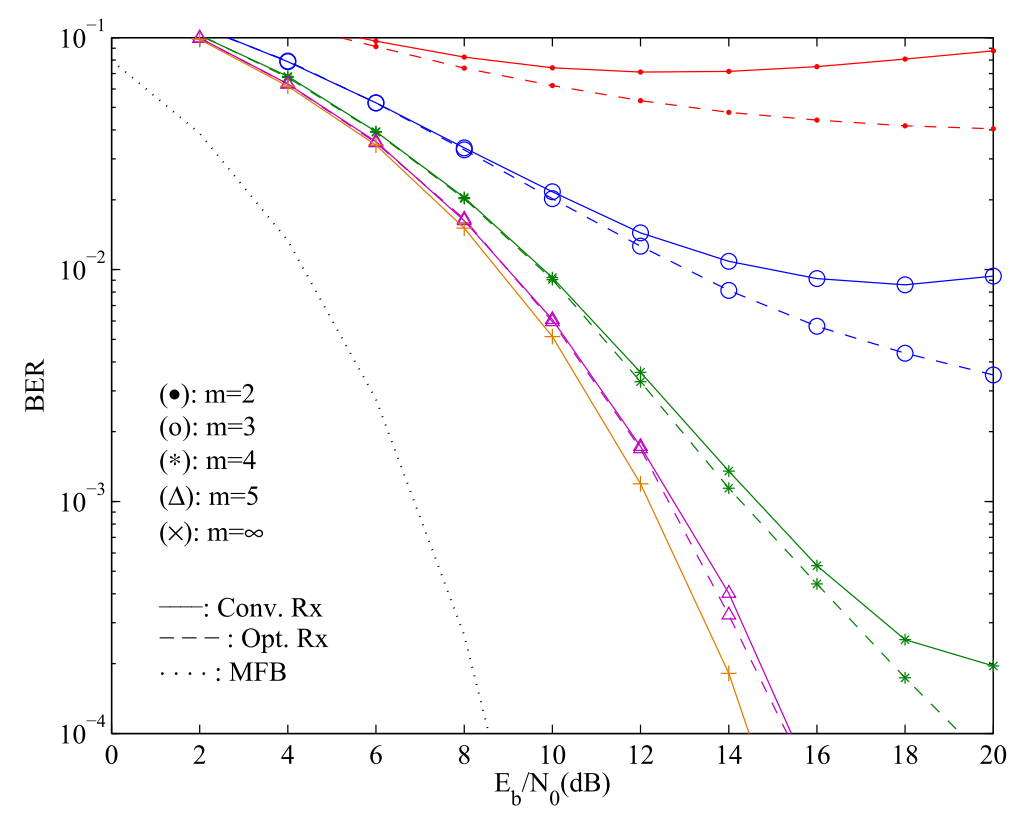

Figure 8 BER performance in a conventional scenario for $P=1 \mathrm{MT}$ and $R=1 \mathrm{BS}$ for the first iteration. With different values of $m$.

show the simulated and its theoretical BER performance results.

As expected, for $m$ high, the quantization effects are negligible, but for low values of $m$, we can have a significant performance degradation when we employ the conventional receivers. With the optimized receiver, we decrease this degradation. Finally, we can see a close match between our theoretical results and the results obtained by simulation, especially for the first iteration, that corresponds to the linear FDE.

Considering now a $\mathrm{BS}$ cooperation scenario with $P=2$ MTs transmitting simultaneously to $R=2 \mathrm{BSs}$, the corresponding BER performance for the first MT, i.e., the MT that is first detected in our SIC-like iterative FDE, and second one, i.e., the MT that is detected in second place, are presented in Figures 12 and 13, respectively. All the

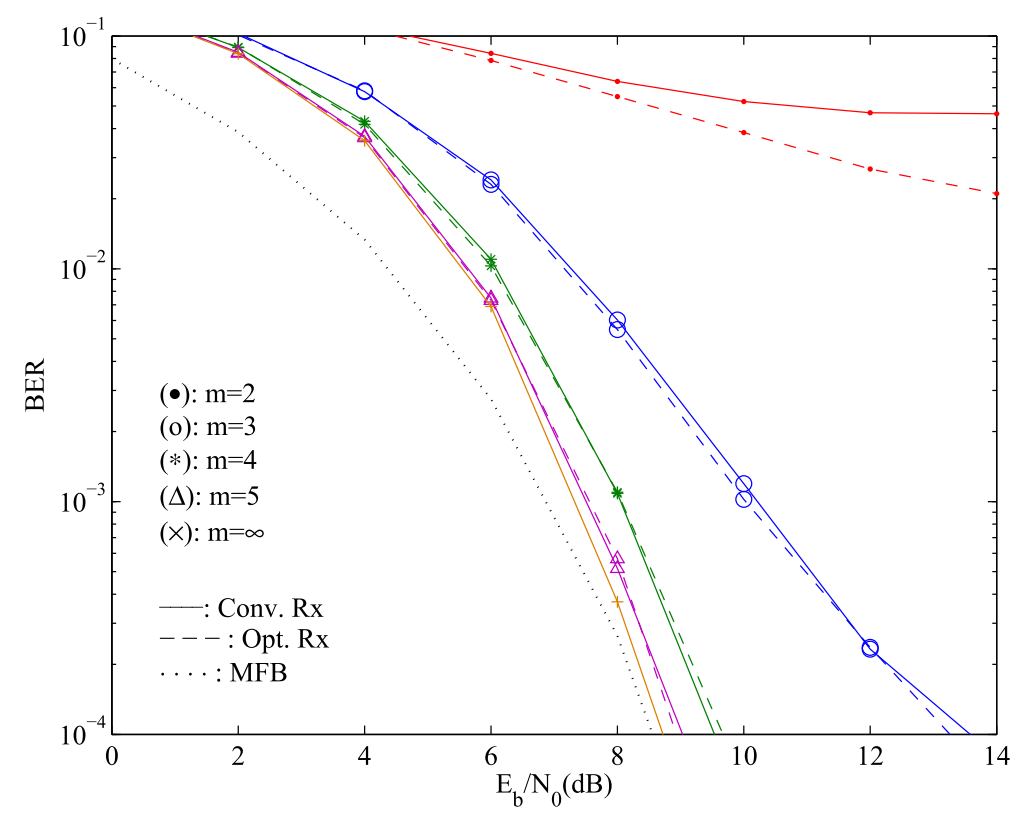

Figure 9 BER performance in a conventional scenario for $P=1 \mathrm{MT}$ and $R=1 \mathrm{BS}$ for the fourth iteration. With different values of $m$. 


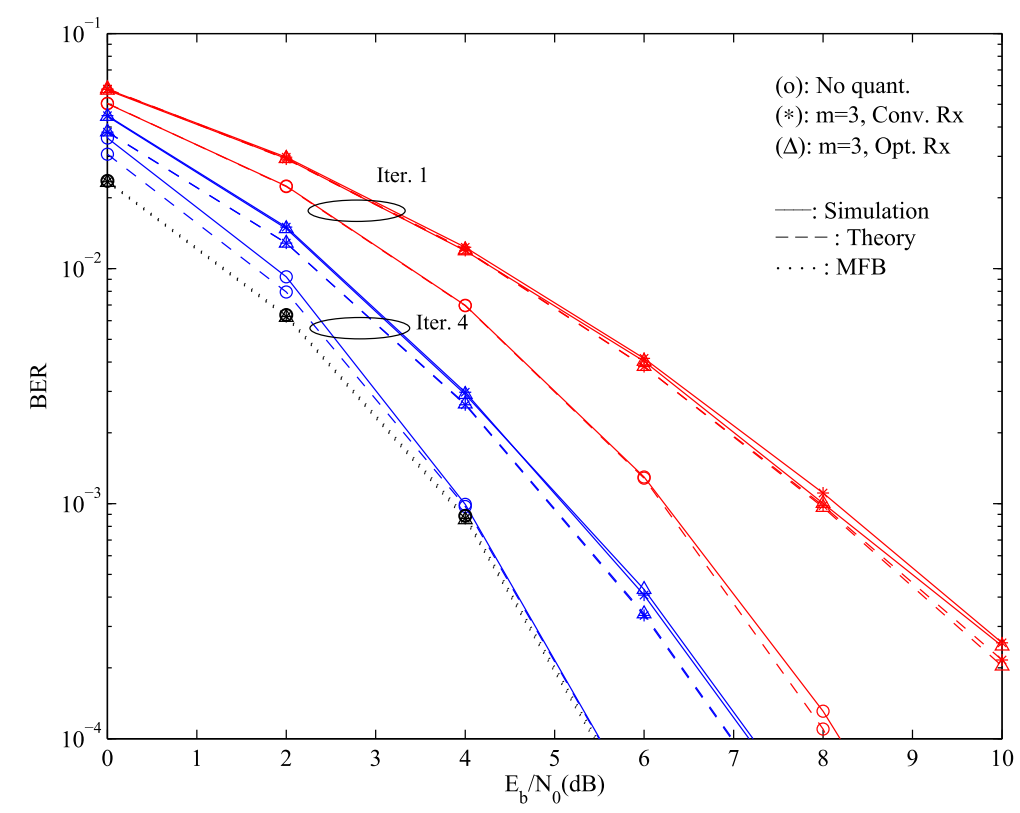

Figure 10 BER performance for $P=1$ in a macro-diversity scenario $(R=2)$ and quantization with $m=3$.

links between the MTs and the BSs are characterized by $\xi_{1,1}=\xi_{1,2}=\xi_{2,1}=\xi_{2,2}=-3 \mathrm{~dB}$.

We considered quantizers with $m=3$ and $m=4$ bits for the real and imaginary parts of the complex envelope samples and the receiver is always designed taking into account the quantization effects. From these figures, it is clear that the proposed receiver is able to separate the MTs. Although the performance for the first MT is significantly worse at the first iteration, the difference between MTs becomes negligible after four iterations, and the resulting performance is already close to the MFB. The degradation due to quantization is slightly higher for this case, especially for the first iteration. Once again, there is a close match between theoretical and simulated results, although the difference is higher than for the case where $P=1$, especially at the fourth iteration. However,

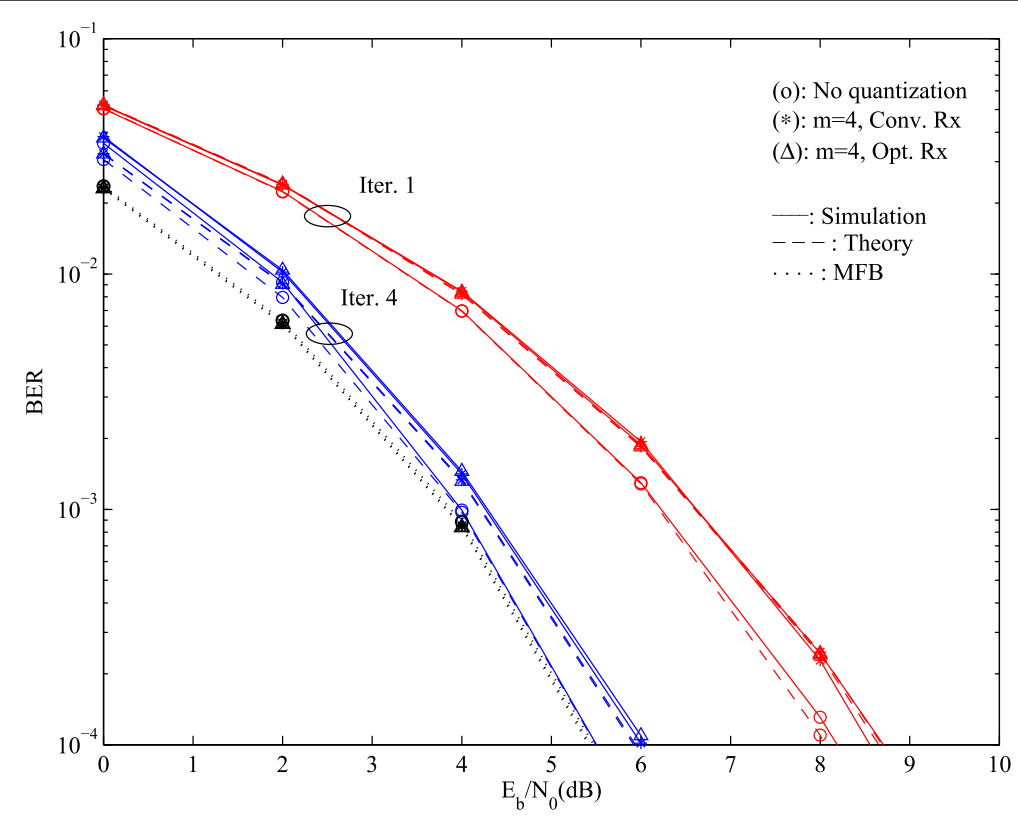

Figure 11 BER performance for $P=1$ in a macro-diversity scenario $(R=2)$ and quantization with $m=4$. 


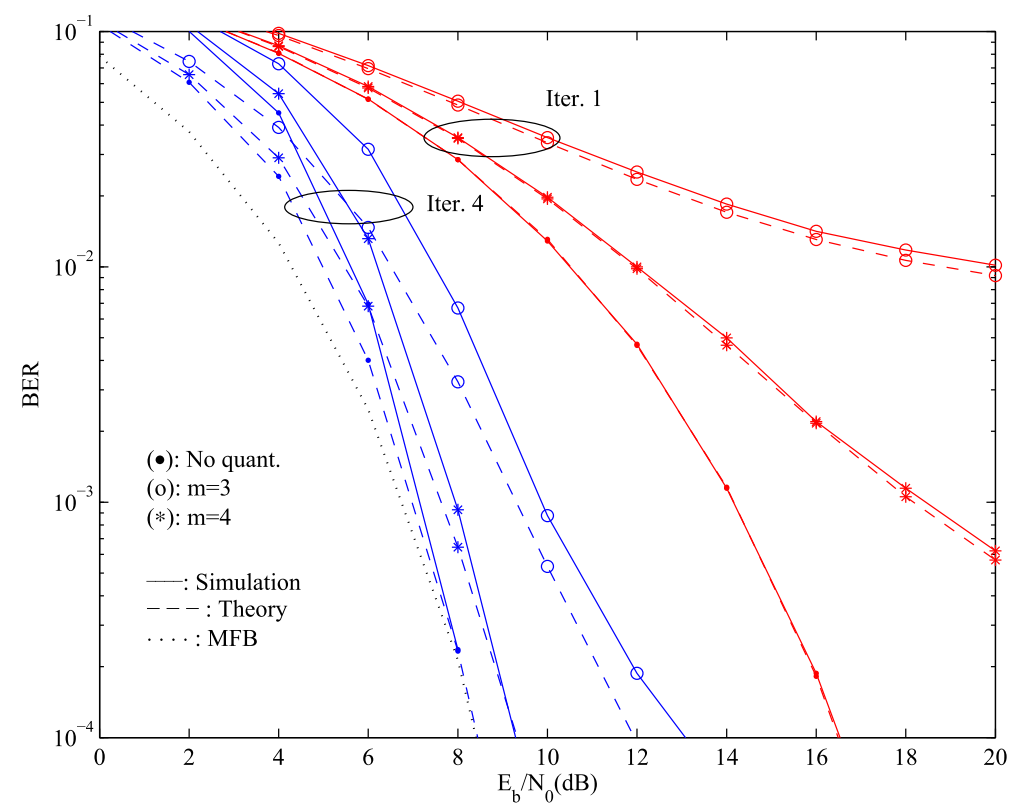

Figure 12 BER performance for a BS cooperation scenario $(P=R=2)$ for the first MT.

the difference is always below $0.5 \mathrm{~dB}$, so our analytical approach is relatively accurate.

Let us consider now a BS cooperation scenario, with $P=2 \mathrm{MTs}$ and $R=2 \mathrm{BSs}$, in which users are not perfectly symmetric and there is not a perfect average power control. Figures 14 and 15 show the simulated and its theoretical BER performance for the first MT and the second
MT, respectively. In both figures, the first and fourth iterations are depicted. The power associated to the different links is given by:

$$
\Xi=\left[\begin{array}{ll}
\xi_{1,1} & \xi_{1,2} \\
\xi_{2,1} & \xi_{2,2}
\end{array}\right]=\left[\begin{array}{cc}
-3 & -3 \\
0 & -10
\end{array}\right](\mathrm{dB})
$$

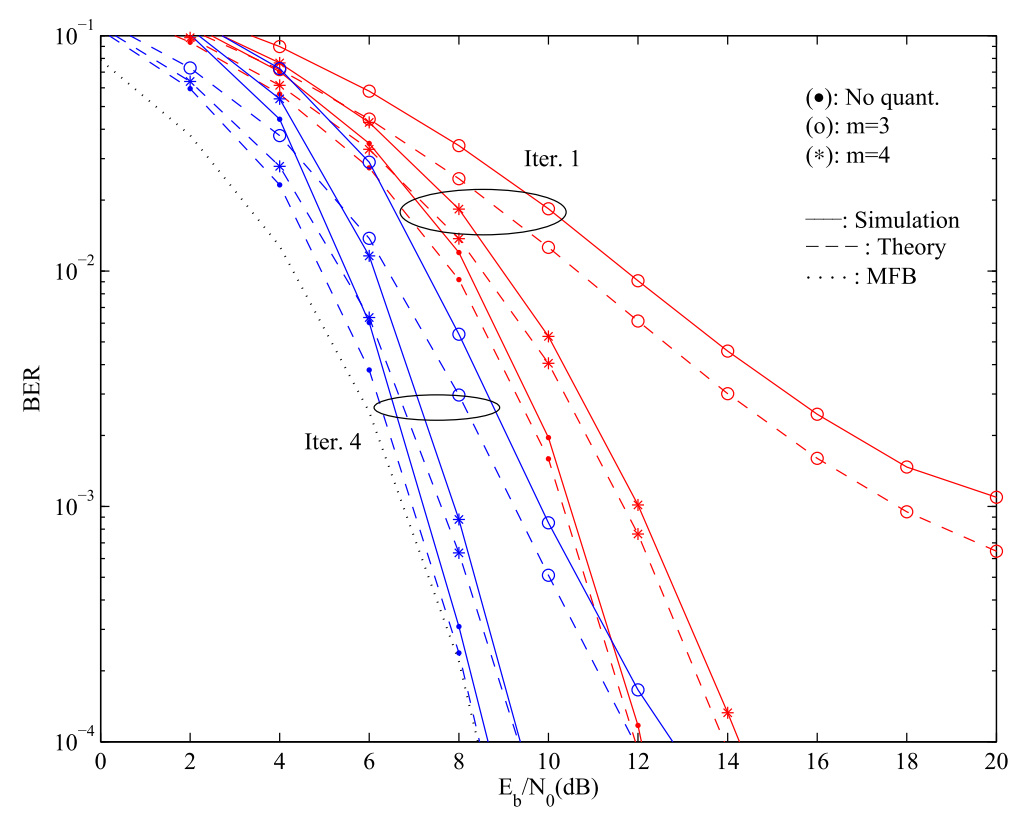

Figure 13 BER performance for a BS cooperation scenario $(P=R=2)$ for the second MT. 


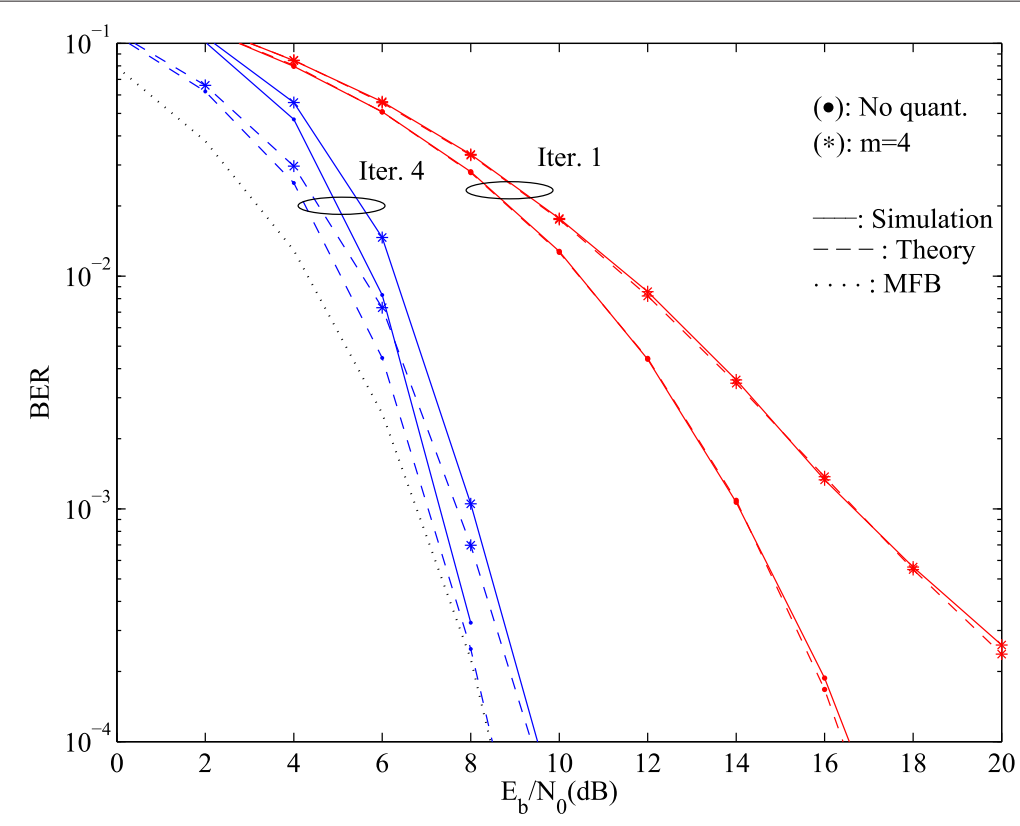

Figure 14 BER performance for a BS cooperation scenario $(P=R=2)$ with users not perfectly symmetric for the first MT.

where the samples are quantized considering an optimized receiver with $m=4$ bits.

This could be regarded as a scenario where one user is between two BSs and the other is closer to one BS.

Finally, let us consider a BS cooperation architecture with $P=3$ MTs transmitting to $R=3$ receiving BSs with all links characterized by $\xi_{p, r}=-4.77 \mathrm{~dB}$ (i.e., $1 / 3$ in linear units), which can be regarded as the case where the MTs are approximately in the intersection of the three cells. The corresponding performance results are depicted in Figure 16.

Clearly, we can still separate the three MTs. Similar results were observed for other values of $P=R$.

As a final remark, we would like to point out that (35) assumes perfect average power control. Our simulations indicate that we are still able to separate the users when we

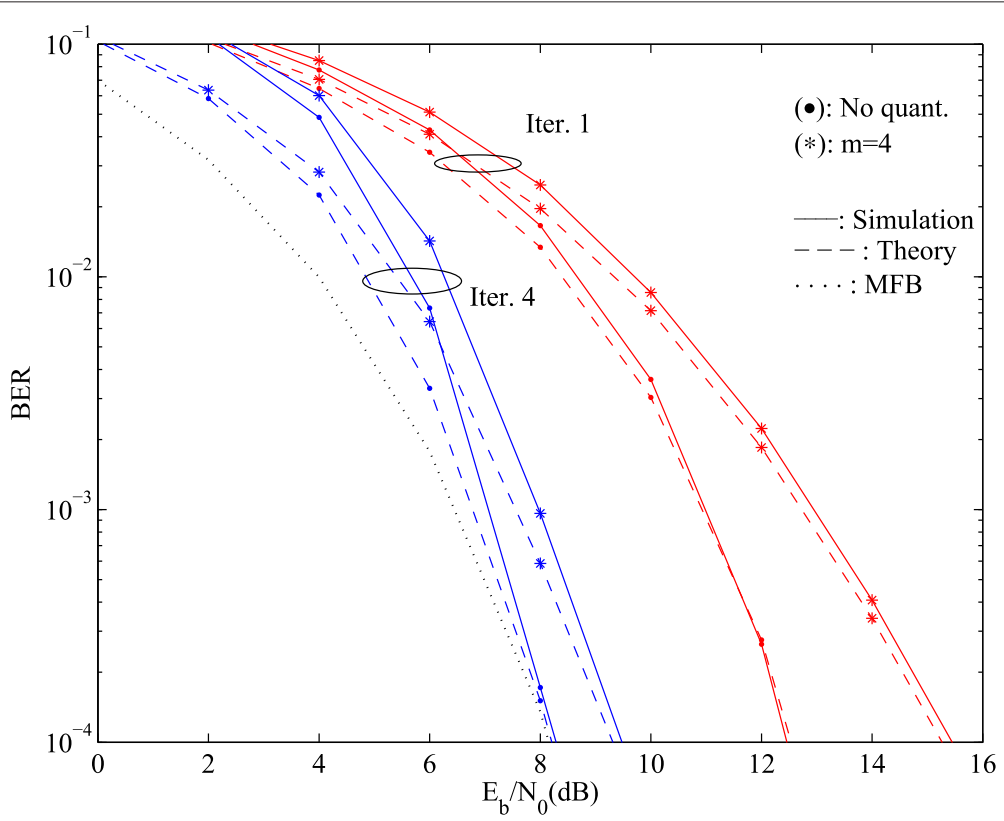

Figure 15 BER performance for a BS cooperation scenario $(P=R=2)$ with users not perfectly symmetric for the second MT. 


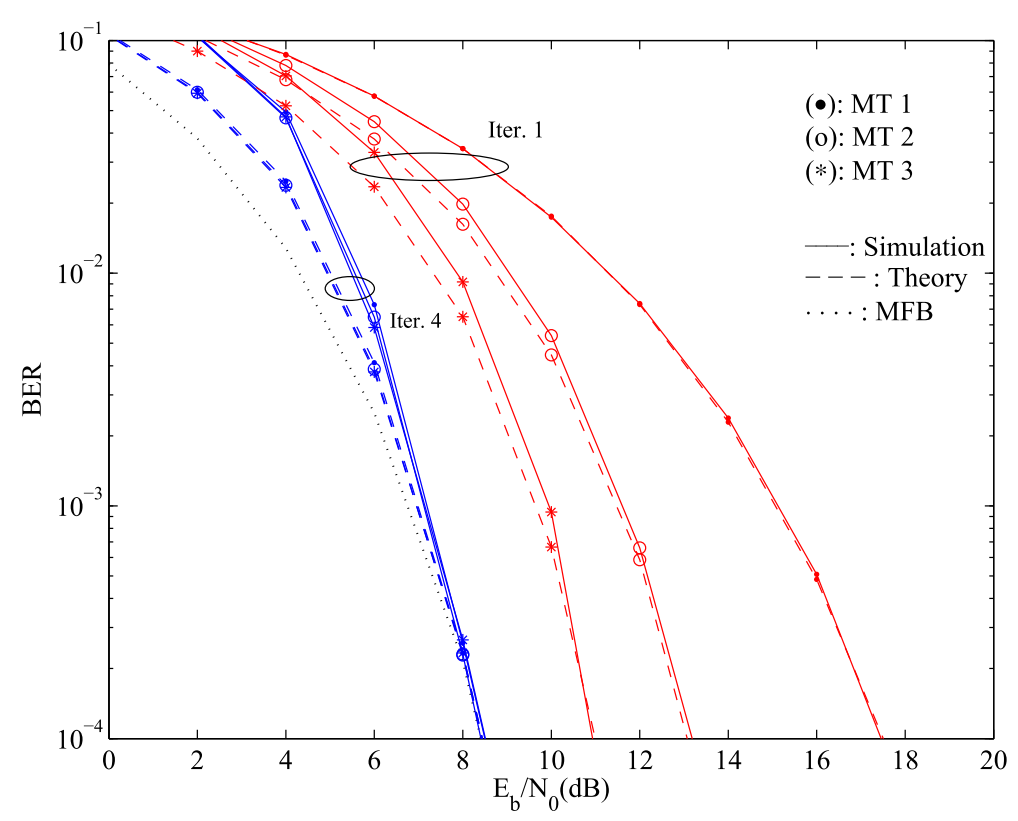

Figure 16 BER performance for a BS cooperation scenario $(P=R=3)$ for iterations 1 and 4 .

do not have power control, i.e., the total received power for distinct users is substantially different.

\section{Conclusions}

In this paper, we considered the receiver design for the uplink transmission in BS cooperation schemes employing SC-FDE signals. Here, the received signals at a certain BS are sampled and have the option of being quantized, with the corresponding bits being transmitted to a central unit that performs the user detection and/or separation using iterative frequency-domain receivers. Also, in this paper, we developed and presented an analytical approach for obtaining the performance of the proposed receivers.

The presented performance results demonstrated that the proposed receivers allow significant macro-diversity gains, as well as an efficient user separation. It is shown that we can have excellent performances using only 4 bits to quantize the real and imaginary parts of the complex envelope of the received signal at the $\mathrm{BS}$, with a performance close to the MFB, and although relatively simple, our analytical approach was shown to be quite accurate. Hence, these techniques are presented as an excellent approach for future broadband wireless systems employing BS cooperation schemes.

\section{Endnotes}

${ }^{a}$ For the sake of simplicity, we will describe only the case where the signals associated with each BS are quantized. The extension for the case where the central unit is placed in a given BS, and we only need to quantize and transmit through the backhaul the signal from the other BS which is straightforward.

${ }^{b}$ Actually, the users should be ordered according to the signal-to-noise plus overall interference ratio (including residual inter-symbol interference (ISI) and residual inter-user interference) at the FDE output, but usually, there is strong correlation between this value and the overall power associated with that MT.

\section{Appendix}

Let us consider a signal $x \sim \mathcal{C N}(0,2)$, i.e., $\operatorname{Re}\{x\} \sim$ $\mathcal{N}(0,1)$ and $\operatorname{Im}\{x\} \sim \mathcal{N}(0,1)$, that is submitted to a given quantizer. It can be shown [34] that $x^{Q}=\alpha x+d$, where $d$ is uncorrelated with $x$, and:

$$
\alpha=\int_{-\infty}^{\infty} \omega f_{Q}(\omega) \frac{1}{\sqrt{2 \pi}} e^{-\frac{\omega^{2}}{2}} d \omega,
$$

with $f_{Q}$ denoting the quantization characteristic associated with the real and imaginary parts of $x$.

Clearly we have:

$$
\begin{gathered}
2 \sigma_{x Q}^{2}=\mathbb{E}\left[\left|x^{Q}\right|^{2}\right]=|\alpha|^{2} \mathbb{E}\left[|x|^{2}\right]+\mathbb{E}\left[|d|^{2}\right]= \\
2|\alpha|^{2}+2 \sigma_{d}^{2}=\int_{-\infty}^{\infty} 2\left|f_{Q}(\omega)\right|^{2} \frac{1}{\sqrt{2 \pi}} e^{-\frac{\omega^{2}}{2}} d \omega .
\end{gathered}
$$

Therefore, the SQNR at the quantizer output is given by:

$$
\mathrm{SQNR}=\frac{\mathbb{E}\left[|\alpha x|^{2}\right]}{\mathbb{E}\left[|d|^{2}\right]}=\frac{2|\alpha|^{2}}{\mathbb{E}\left[|d|^{2}\right]}=\frac{|\alpha|^{2}}{\sigma_{d}^{2}},
$$

where $\sigma_{d}^{2}=\sigma_{x Q}^{2}-|\alpha|^{2}$ that is obtained from (37) and (38). 


\section{Competing interests}

The authors declare that they have no competing interests.

\section{Acknowledgements}

The authors would like to thank the anonymous reviewers for their values comments which helped improve the paper. This work was partially supported by the FCT (Fundação para a Ciência e a Tecnologia) under projects PEst-OE/EEI/LA0008/2013, HETCOP, ADIN (PTDC/EEI- TEL/2990/2012), CoPWIN (PTDC/EEI- TEL/1417/2012) and grant SFRH/BD/87524/2012.

\section{Author details}

${ }^{1}$ IT - Instituto de Telecomunicações, 1049-001 Lisboa, Portugal. ${ }^{2}$ ISCTE Instituto Universitário de Lisboa, 1649-026 Lisboa, Portugal. ${ }^{3} \mathrm{FCT}$ Universidade Nova de Lisboa, 2829-516 Caparica, Portugal. ${ }^{4}$ DETI Universidade de Aveiro, 3810-193 Aveiro, Portugal.

Received: 3 June 2014 Accepted: 28 December 2014

Published online: 21 January 2015

\section{References}

1. M Dohler, RW Heath, A Lozano, CB Papadias, RA Valenzuela, Is the PHY layer dead? IEEE Commun. Mag. 49(4), 159-165 (2011)

2. C Shannon, A Mathematical theory of communication. Bell Syst. Tech. J. 27, 379-423623656 (1948)

3. D Gesbert, S Hanly, H Huang, S Shamai Shitz, O Simeone, W Yu, Multi-cel MIMO cooperative networks: a new look at interference. IEEE J. Select. Areas Commun. 28(9), 1380-1408 (2010)

4. J Zhang, R Chen, JG Andrews, A Ghosh, RW Heath, Networked MIMO with clustered linear precoding. Wireless Commun. IEEE Trans. 8(4), 1910-1921 (2009)

5. M Sawahashi, Y Kishiyama, A Morimoto, D Nishikawa, M Tanno, Coordinated multipoint transmission/reception techniques for LTE-advanced [coordinated and distributed MIMO]. Wireless Commun. IEEE. 17(3), 26-34 (2010)

6. X-H You, D-M Wang, B Sheng, X-Q Gao, X-S Zhao, M Chen, Cooperative distributed antenna systems for mobile communications [coordinated and distributed MIMO]. Wireless Commun. IEEE. 17(3), 35-43 (2010)

7. $\mathrm{H}-\mathrm{H}$ Choi, On the degrees of freedom of interference alignment for multicell MIMO interfering broadcast channels. Sci. World J.

2014, 1-8 (2014)

8. 3GPP Long Term Evolution. http://www.3gpp.org/LTE

9. FC Ribeiro, R Dinis, F Cercas, A Silva, in Computer Communications and Networks (ICCCN) 201221st International Conference On. Iterative frequency-domain receivers for the uplink of cellular systems with base station cooperation (Munich, Germany, 2012)

10. O Somekh, BM Zaidel, S Shamai, Sum rate characterization of joint multiple cell-site processing. IEEE Trans. Inform. Theory. 53(12), 4473-4497 (2007)

11. E Bjol andrnson, R Zakhour, D Gesbert, B Ottersten, Cooperative multicell precoding: rate region characterization and distributed strategies with instantaneous and statistical CSI. IEEE Trans. Signal Process. 58(8) , 4298-4310 (2010)

12. A Silva, R Holakouei, D Castanheira, A Gameiro, R Dinis, A novel distributed power allocation scheme for coordinated multicell systems. J. Wireless Commun. Netw. EURASIP. 30, 1-11 (2013)

13. L Cimini, Analysis and simulation of a digital mobile channel using orthogonal frequency division multiplexing. Commun. IEEE Trans. 33(7), 665-675 (1985)

14. H Sari, G Karam, I Jeanclaude, in Vehicular Technology Conference, 1994 IEEE 44th. An analysis of orthogonal frequency-division multiplexing for mobile radio applications (Stockholm, Sweden, 1994), pp. 1635-16393

15. A Gusmao, R Dinis, J Conceicao, N Esteves, in Vehicular Technology Conference Proceedings, 2000. VTC 2000-Spring Tokyo 2000 IEEE 51st. Comparison of two modulation choices for broadband wireless communications, vol. 2 (Tokyo, Japan, 2000), pp. 1300-13052

16. D Falconer, SL Ariyavisitakul, A Benyamin-Seeyar, B Eidson, Frequency domain equalization for single-carrier broadband wireless systems. IEEE Commun. Mag. 40(4), 58-66 (2002)

17. R Kalbasi, R Dinis, D Falconer, A Banihashemi, in Acoustics, Speech, and Signal Processing, 2004. Proceedings. (ICASSP'04). IEEE International Conference On. An iterative frequency-domain layered space-time receiver for SDMA systems with single-carrier transmission, vol. 4 (Montreal, Canada, 2004), pp. 793-7964

18. R Dinis, R Kalbasi, D Falconer, AH Banihashemi, Iterative layered space-time receivers for single-carrier transmission over severe time-dispersive channels. IEEE Commun. Lett. 8(9), 579-581 (2004)

19. N Benvenuto, R Dinis, D Falconer, S Tomasin, Single carrier modulation with nonlinear frequency domain equalization: an idea whose time has come again. Proc. IEEE. 98(1), 69-96 (2010)

20. M Tüchler, R Koetter, AC Singer, Turbo equalization: principles and new results. IEEE Trans. Commun. 50(5), 754-767 (2002)

21. M Tüchler, J Hagenauer, in Allerton Conf. Turbo equalization using frequency domain equalizers (Monticello, USA, 2000)

22. M Tüchler, J Hagenauer, in IEEE Vehicular Technology Conference, 2001. VTC 2001 Fall. Linear time and frequency domain turbo equalization, vol. 4 (2001), pp. 2773-2777

23. N Benvenuto, $\mathrm{S}$ Tomasin, Iterative design and detection of a DFE in the frequency domain. IEEE Trans. Commun. 53(11), 1867-1875 (2005)

24. C-T Lam, DD Falconer, F Danilo-Lemoine, R Dinis, in Vehicular Technology Conference, 2006. VTC-2006 Fall 2006 IEEE 64th. Channel estimation for SC-FDE systems using frequency domain multiplexed pilots (Montreal, Canada, 2006), pp. 1-5

25. F Coelho, R Dinis, P Montezuma, in Military communications conference, 2010 - MILCOM 2010. Joint detection and channel estimation for block transmission schemes, (2010), pp. 1765-1770. doi:10.1109/MILCOM. 2010.5680240

26. F Coelho, R Dinis, P Montezuma, in Vehicular Technology Conference (VTC Fall) 2011, IEEE. Efficient channel estimation for single frequency broadcast systems (San Francisco, USA, 2011), pp. 1-6

27. N Souto, R Dinis, JC Silva, Impact of channel estimation errors on SC-FDE systems. Commun. IEEE Trans. 62(5), 1530-1540 (2014)

28. F Silva, R Dinis, PM Montezuma, Estimation of the feedback reliability for IB-DFE receivers. ISRN Commun. Netw. J. 2011, 1-7 (2011)

29. FC Ribeiro, R Dinis, F Cercas, A Silva, in Globecom Workshops (GC Wkshps) 2012 IEEE. Analytical performance evaluation of Base Station cooperation systems using SC-FDE modulations with iterative receivers (Anaheim, USA, 2012), pp. 637-641

30. A Gusmao, P Torres, R Dinis, N Esteves, A turbo FDE technique for reduced-CP SC-based block transmission systems. IEEE Trans. Commun. 55(1), 16-20 (2007)

31. A Gusmao, P Torres, R Dinis, N Esteves, in Turbo Codes Related Topics; 6th International ITG-Conference on Source and Channel Coding (TURBOCODING), 20064 th International Symposium On. A class of iterative FDE techniques for reduced-CP SC-based block transmission (Munich, Germany, 2006), pp. 1-6

32. R Dinis, N Esteves, in Proc. Wireless. On broadband block transmission over strongly frequency-selective fading channels (Calgary, Canada, 2003)

33. T Araujo, R Dinis, Performance evaluation of quantization effects on multicarrier modulated signals. IEEE Trans. Vehic. Technol. 56(5), 2922-2930 (2007)

34. HE Rowe, Memoryless nonlinearities with Gaussian inputs: elementary results. Bell Syst. Tech. J. 61(7), 1519-1525 (1982)

\section{Submit your manuscript to a SpringerOpen ${ }^{\circ}$ journal and benefit from:}

- Convenient online submission

- Rigorous peer review

- Immediate publication on acceptance

- Open access: articles freely available online

- High visibility within the field

- Retaining the copyright to your article

Submit your next manuscript at $>$ springeropen.com 